\title{
Paper
}

\section{Economic Evaluation of DC Smart House Groups Considering Electric Vehicle Operation Patterns}

\author{
Kentaro Shimomachi $^{* \dagger}$ Member, $\quad$ Yuji Mishima ${ }^{\dagger}$ Member
}

(Received November 30, 2020, revised January 16, 2021)

\begin{abstract}
Smart houses, which have installed on-site distributed generators such as photovoltaics and batteries and manage their own energy usage using information technology, are being studied and developed around the world. Most of the facilities in a smart house are operated as DC systems; however, conventional supply systems are AC. Moreover, the number of electric vehicles (EV), which also need DC power, have been increasing on the residential side. Therefore, a DC power supply system for smart houses can reduce the number of power converters, conversion losses, and electric charges. The initial and running costs of DC- and AC-based smart houses were calculated under various conditions in this paper. It was concluded that DC-based smart houses have lower costs than AC-based ones regardless of the AC/DC ratio in the load and in the use of EV.
\end{abstract}

Keywords: Smart house, DC power supply, Cost reduction,

\section{Introduction}

In recent years, an increasing number of studies have aimed to disseminate renewable energy (RE), such as photovoltaics (PV) and wind energy conversion systems. Most of the output of RE generation fluctuates owing to the effects of weather and time, etc. The installation of an energy storage system (ESS) such as a battery is one method of controlling RE outputs [1]. By expanding this method, the use of smart grids, which can correct and process information from facilities, namely RE output data, ESS data, and load data is becoming increasingly common [2] [3]. Moreover, the idea of a smart house (SH), which applies this idea, not only to a power system but also to individual houses, is also increasing. For example, a home energy management system (HEMS) is proposed in [4], and it can enhance the energy efficiency in an $\mathrm{SH}$.

On the other hand, conventional power systems supply AC power to meet demands. Therefore, power converters are necessary for PVs and ESSs to interconnect power systems because they output DC power. On the demand side, many electric appliances consume DC power. Therefore, outputs from a DC source must go through DC/AC and $\mathrm{AC} / \mathrm{DC}$ converters, even if the demands need DC power only. This fact illustrates that it is uneconomical to directly install a DC source for DC loads. In [5], reductions in the number of converters and the amount of conversion losses were achieved by installing a DC power supply system at a data center that had a greater amount of DC loads than residences. Moreover, a high-efficiency energy supply method for an SH with only DC loads is proposed in [6]. In a case

\footnotetext{
* Corresponding:shimomachi@hakodate-ct.ac.jp

†ational Institute of Technology(KOSEN), Hakodate College

14-1, Tokura-cho, Hakodate-shi, Tokyo, Japan 042-8501
}

in which mixed AC and DC loads remained in the $\mathrm{SH}$, it was shown that the ratio of DC loads could determine the cost reduction in [7]. Also, electric vehicles (EV), which also use DC power, are seeing increased use on the residential side. Therefore, DC power supplies in SHs will be required to achieve high-efficiency energy use in the future. In [7], two types of multiple $\mathrm{SH}$ connection are discussed: SHs connected independently to conventional AC grids and small DC networks that contain SHs connected to grids are evaluated from an economic point of view. PVs and ESSs are considered in [7]; however, EVs are not considered. Therefore, economic evaluation of power supply forms in SHs that include EV operation patterns is proposed in this paper.

\section{Outline of an SH Group}

2.1 Structure of an SH group In this paper, it is assumed that five SHs make a group and that they are connected to the same feeder line, as shown in Fig. 1 and Fig. 2. Also in this paper, a group in which each $\mathrm{SH}$ is independently connected to an AC line is called an ACSH group, and a small DC network that contains SHs is called a DCSH group. In both cases, every $\mathrm{SH}$ has an AC load, DC load, $\mathrm{PV}, \mathrm{EV}$, and battery. Although the electricity from each $\mathrm{SH}$ can flow into the utility grid in principle, reverse power flow from SHs is inhibited to avoid the situation in which SHs earn an electricity benefit by exploiting time-of-use (TOU) costs. The capacities of PVs and batteries are determined by solving the optimization problems shown in the next section.

2.2 Problem formulation In this paper, the capacities of PVs, batteries, and interfacing converters are optimized to minimize the total cost, including the initial and running costs in SH groups. Therefore, the objective func- 


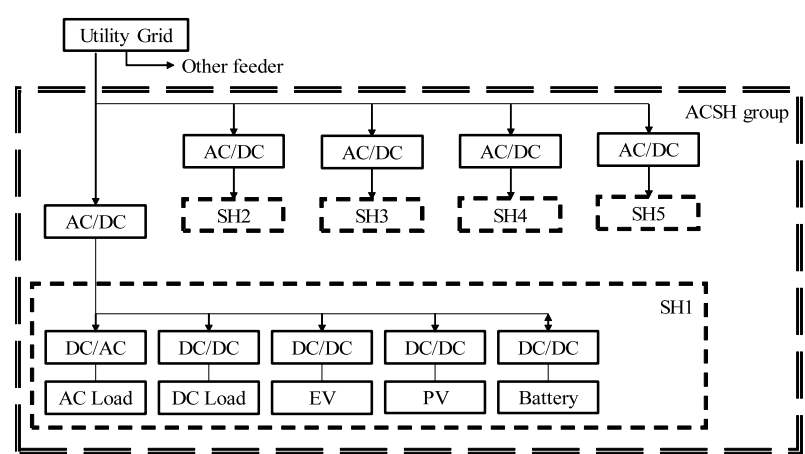

Figure 1: Structure of ACSH group.

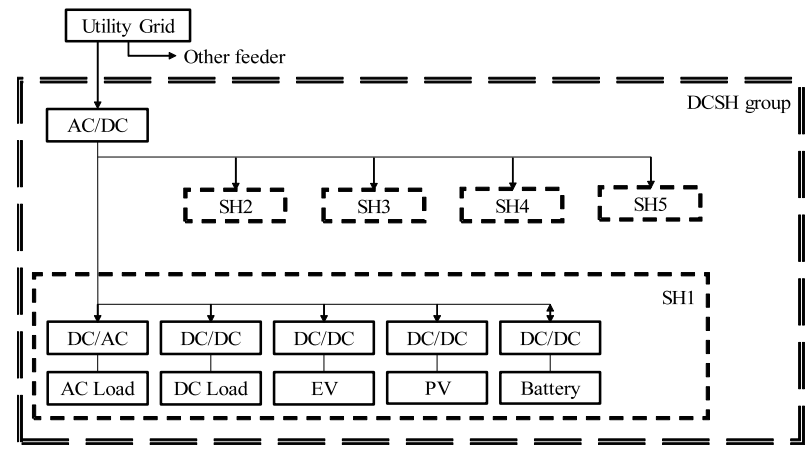

Figure 2: Structure of DCSH group.

tion in this problem can be written as

$$
\operatorname{minf}=I C_{P V}+I C_{b a t t}+I C_{i n t}+R C
$$

where each IC represents the initial cost of each facility [JPY], namely, PVs, batteries, and interfacing converters, and $R C$ is the running cost of SH groups during a day [JPY]. In particular, each cost can be written as

$$
\begin{aligned}
& I C_{P V}=\sum_{n=1}^{5} C_{P V} \cdot C a p_{P V}^{n} \\
& I C_{\text {batt }}=\sum_{n=1}^{5} C_{\text {batt }} \cdot \text { Cap }_{\text {batt }}^{n} \\
& I C_{\text {conv }}=\sum_{n=1}^{5} C_{\text {conv }} \cdot \text { Cap ponv }_{\text {con }}^{n} \\
& R C=\sum_{n=1}^{5} \sum_{t=1}^{24} \operatorname{TOU}(t) \cdot P_{\text {grid }}^{n}(t) \cdot \Delta T
\end{aligned}
$$

where each $C$ is the unit initial cost $[\mathrm{JPY} / \mathrm{kW}]$ and each $C a p$ is the capacity of the facility in the $n$-th $\mathrm{SH}[\mathrm{kW}]$. In the case of the ACSH group, $I C_{\text {conv }}$ must be written in summation form, as in Eq. 4, because each $\mathrm{SH}$ has its own interfacing converter. Also, $\operatorname{TOU}(t)$ is the electricity price at time $t[\mathrm{JPY} / \mathrm{kWh}], P_{\text {grid }}^{n}(t)$ is the purchasing power of the $n$-th SH from the utility grid (in the case of an ACSH group) [kW], $P_{\text {grid }}(t)$ is the purchasing power of the DCSH group [kW], and $\Delta T$ is the duration in the numerical simulation [h]. In this paper, a day is partitioned into 24 durations and $\Delta T$ must be 1.0 [h]. In the case of a DCSH group, an interfacing converter is integrated. Therefore, $I C_{\text {conv }}$ and $R C$ can be written as Eq. $4^{\prime}$ and Eq. 5', respectively.

$$
\begin{gathered}
I C_{\text {conv }}=C_{\text {conv }} \cdot \text { Cap }_{\text {conv }} \\
R C=\sum_{T=1}^{24} T O U(t) \cdot P_{\text {grid }}(t) \cdot \Delta T
\end{gathered}
$$

The constraints on solving the problems are written as follows.

(a) Power balance constraint

The summation of the supply power flow from the grid and/or facilities must equal the load, which may include the charging power of the EVs and batteries. This constraint must be satisfied for any duration and $\mathrm{SH}$.

$$
\begin{aligned}
& P_{g r i d}^{n}(t)+P_{P V}^{n}(t)+P_{b a t t_{D}}^{n}(t) \\
= & P_{A C L}^{n}(t)+P_{D C L}^{n}(t)+P_{E V_{C}}^{n}(t)+P_{\text {batt }_{C}}^{n}(t)
\end{aligned}
$$

Here, $P^{n}(t)$ is the power flow of each facility [kW], and the suffixes batt $_{C}$ and batt $_{D}$ indicate charging and discharging, respectively. This constraint can be rewritten in the case of a DCSH group because the reverse power flow from one $\mathrm{SH}$ to another is not inhibited. Therefore, the constraint is applied to the network, under the interfacing converter. This means that the power flow from each $\mathrm{SH}$ can be collected as follows:

$$
\begin{aligned}
& P_{\text {grid }}(t)+P_{P V}^{n}(t)+P_{\text {batt }_{D}}^{n}(t) \\
= & \sum_{n=1}^{5} P_{A C L}^{n}(t)+P_{D C L}^{n}(t)+P_{E V_{C}}^{n}(t)+P_{\text {batt }_{C}}^{n}(t)
\end{aligned}
$$

(b) Capacity constraint

The power flow of each facility cannot exceed its own $\mathrm{kW}$ capacity limitation.

$$
\begin{aligned}
& P_{x x}(t) \leq P_{x x}^{\max } \\
& P_{x x}(t) \geq P_{x x}^{\min }
\end{aligned}
$$

Here, the suffix $x x$ represents the facility, and min and max indicate the lower and upper limits, respectively.

(c) Battery charging and discharging constraint

In general, a battery cannot charge and discharge simultaneously. To avoid such a situation in the simulations, binary (0-1) variables, which indicate the state of the battery, are introduced. Therefore, the capacity constraints of a battery should be rewritten as follows:

$$
\begin{aligned}
& P_{\text {batt }}^{\min } \leq P_{\text {batt }_{C}}^{n}(t) \leq P_{\text {batt }}^{\max } \cdot\left\{1-u_{\text {batt }}^{n}(t)\right\} \\
& P_{\text {batt }}^{\min } \leq P_{\text {batt }}^{n}(t) \leq P_{\text {batt }}^{\text {max }} \cdot u_{\text {batt }}^{n}(t)
\end{aligned}
$$

If $u_{b a t t}^{n}(t)=0$, the battery can charge but cannot discharge, and vice versa. 
(d) Constraint concerning state of charge (SOC)

The SOC of a battery depends on its previous state. The units are $\mathrm{kWh}$.

$$
\begin{aligned}
& S O C_{\text {batt }}^{n}(t+1) \\
= & S O C_{\text {batt }}^{n}(t)+\left\{P_{\text {batt }_{C}}^{n}(t)-P_{\text {batt }_{D}}^{n}(t)\right\} \cdot \Delta T
\end{aligned}
$$

On the other hand, EVs have also their own batteries. Therefore, the SOC of an EV can be written similarly, as follows.

$$
\begin{aligned}
& \operatorname{SOC}_{E V}^{n}(t+1) \\
= & S O C_{E V}^{n}(t)+\left\{P_{E V_{C}}^{n}(t)-P_{E V_{D}}^{n}(t)\right\} \cdot \Delta T
\end{aligned}
$$

However, it is assumed that EVs cannot discharge their SOC for energy supply. Therefore, $P_{E V_{D}}(t)$ is used only for driving in this paper. Both SOCs are limited between the lower and upper limits at any time.

$$
\begin{aligned}
& S O C_{\text {batt }}^{n}(t) \leq S O C_{\text {batt }}^{n \max } \\
& S O C_{\text {batt }}^{n}(t) \geq S O C_{\text {batt }}^{n \text { min }} \\
& S O C_{E V}^{n}(t) \leq S O C_{E V}^{n \max } \\
& S O C_{E V}^{n}(t) \geq S O C_{E V}^{n \min }
\end{aligned}
$$

In general, the upper limit of an SOC depends on its $\mathrm{kW}$ capacity (2.2 times in this paper).

(e) $\mathrm{CO}_{2}$ emissions reduction constraint

One of effects of the introduction of SHs is $\mathrm{CO}_{2}$ emissions reduction. The government of Japan has set a $\mathrm{CO}_{2}$ emissions reduction goal of $26 \%$, referenced on 2013. Therefore, SHs should contribute to this reduction, not only for their own aim. Here, it is assumed that $\mathrm{SHs}$ should achieve $\mathrm{CO}_{2}$ emissions reductions as follows:

$$
\frac{\mathrm{CO}_{2}^{\text {base }}-\mathrm{CO}_{2}}{\mathrm{CO}_{2}^{\text {base }}} \geq 0.26
$$

Here, $\mathrm{CO}_{2}^{\text {base }}$ is a referenced value $[\mathrm{kg}]$ and $\mathrm{CO}_{2}$ is the calculated $\mathrm{CO}_{2}$ emissions from the SHs [kg]. Eq. 18 is for the ACSH group, and Eq. 18' is for the DCSH group.

$$
\begin{aligned}
& \mathrm{CO}_{2}=\sum_{n=1}^{5} \sum_{t=1}^{24} C O_{2}^{\text {unit }}(t) \cdot P_{\text {grid }}^{n}(t) \\
& C O_{2}=\sum_{t=1}^{24} C O_{2}^{\text {unit }}(t) \cdot P_{\text {grid }}(t)
\end{aligned}
$$

Finally, the decision variables in this problem are the following: $C_{a p}^{n}, C_{P a p_{b a t t}^{n}}^{n}$, either $C_{a} p_{c o n v}^{n}$ or Cap conv , either $P_{\text {grid }}^{n}(t)$ or $P_{\text {grid }}(t), P_{\text {batt }_{C}}^{n}(t), P_{\text {batt }}^{n}(t)$, and $u_{b a t t}^{n}(t)$. This is a 0-1 linear problem because all of the decision variables except $u_{b a t t}^{n}(t)$ are real numbers and all constraints are linear.

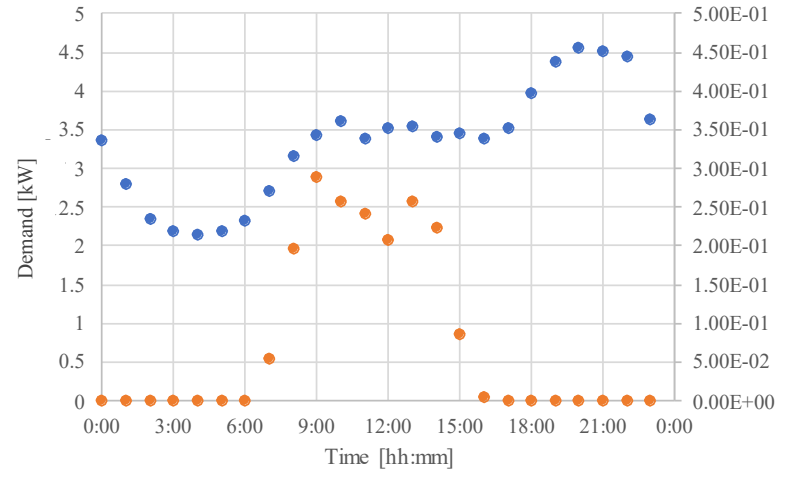

Figure 3: Demand PV data.

Table 1: Given data in a simulation.

\begin{tabular}{cc}
\hline Character & Value \\
\hline$C_{P V}$ & 100 \\
$C_{\text {batt }}$ & 100 \\
$C_{\text {conv }}$ & 20 \\
$T O U(t)$ & $28.08[\mathrm{JPY} / \mathrm{kWh}](7: 00-23: 00)$ \\
& $14.13[\mathrm{JPY} / \mathrm{kWh}]($ Other $)$ \\
$C O_{2}^{\text {unit }}(t)$ & $0.690[\mathrm{~kg} / \mathrm{kWh}](6: 00-18: 00)$ \\
$P_{E V}^{\min }$ & $0.469[\mathrm{~kg} / \mathrm{kWh}]($ Other $)$ \\
$P_{E V}^{\max }$ & $0.0[\mathrm{~kW}]$ \\
$S O C_{E V}^{\min }$ & $3.0[\mathrm{~kW}]$ \\
$S O C_{E V}^{\max }$ & $0[\mathrm{kWh}]$ \\
\hline
\end{tabular}

\section{Simulations}

To evaluate the ACSH and DCSH groups, a summer day is employed for numerical simulation. In this paper, two cases are simulated. First, the ratio of the DC load is changed from $0 \%$ to $100 \%$. This simulation does not include EVs. Then, another case, which considers various EV operation patterns for a fixed DC load ratio, is simulated. The given data for both simulations are shown in Table 1. These same values were used in [7]. The demand data and PV output unit data are shown in Fig. 3.

3.1 Effects of ratio of DC The total costs of the ACSH and DCSH groups are shown in Fig. 4. The ratio of DC load is on the horizontal axis. At every ratio, the DCSH group is lower than the ACSH group. Especially, in case of $40 \%$, the cost gap becomes largest with a value of 4,913 [JPY]. The installed capacities in the case of $40 \%$ are shown in Table 2. The output of each facility is shown in Fig. 5 and Fig. 6. The change in SOC for each SH group is shown in Fig. 7. First, the capacity of the battery of a DCSH is smaller than that of an ACSH. Then, the SOC of the battery reaches the upper limit during daytime. The PV outputs are greater than the demand in each case owing to $\mathrm{CO}_{2}$ emissions reduction constraints. Therefore, the surplus power must be absorbed by the battery or shared to another SH. However, the ACSH group cannot choose to share power and the battery must be large. 


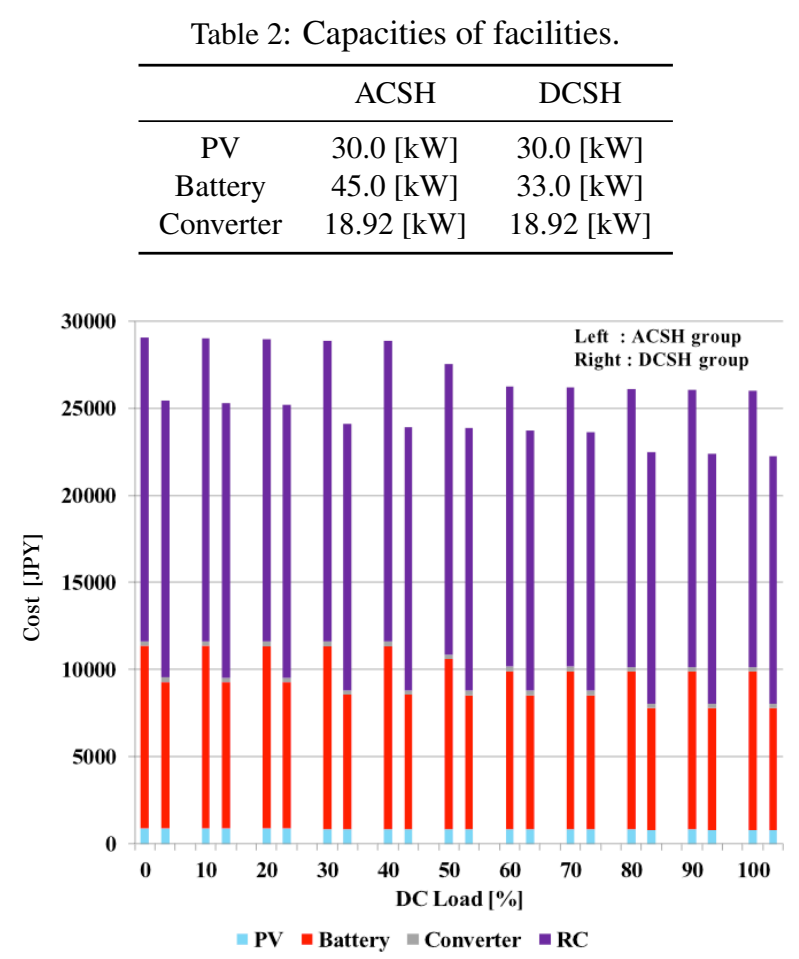

Figure 4: Total cost comparison between ACSG and DCSH.

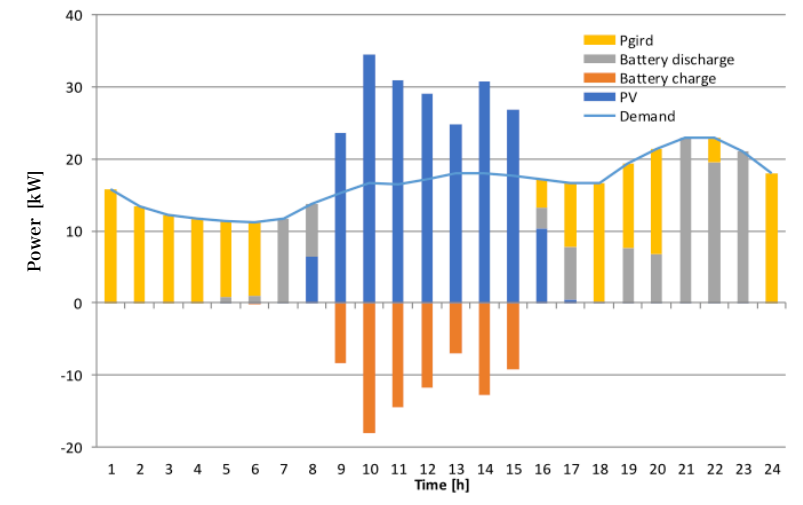

Figure 5: Operation of ACSH group.

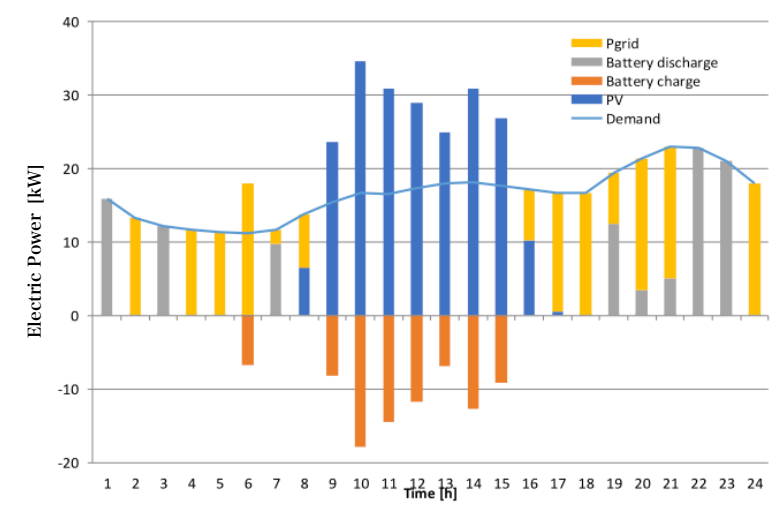

Figure 6: Operation of DCSH group.

3.2 Effects of EV operation patterns This section describes the effects of EV operation patterns on the simulation results. In all of the simulations, the DC load ratio

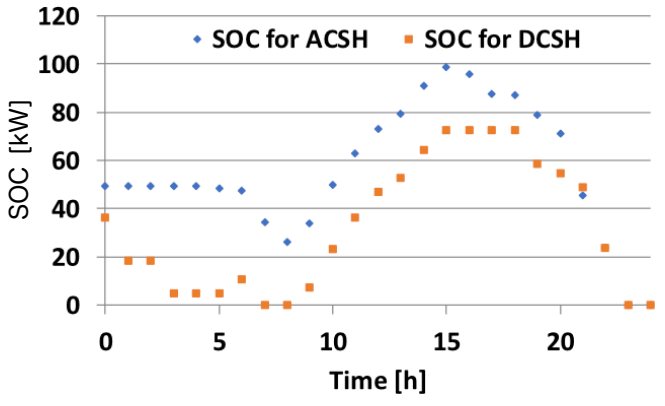

Figure 7: Change in SOC.

Table 3: EV operation patterns.

\begin{tabular}{ccccccc}
\hline $\begin{array}{c}\text { Pattern } \\
\text { No. }\end{array}$ & Category & \multicolumn{5}{c}{ Purpose } \\
\hline 1 & No EV & - & - & - & - & - \\
2 & All for A & A & A & A & A & A \\
3 & All for B & B & B & B & B & B \\
4 & All for C & C & C & C & C & C \\
5 & Varying & C & - & - & - & - \\
6 & & C & C & - & - & - \\
7 & & C & C & C & - & - \\
8 & & C & C & C & C & - \\
9 & Town & A & A & A & B & B \\
10 & City & A & A & B & B & C \\
11 & Metropolis & A & A & B & B & - \\
\hline
\end{tabular}

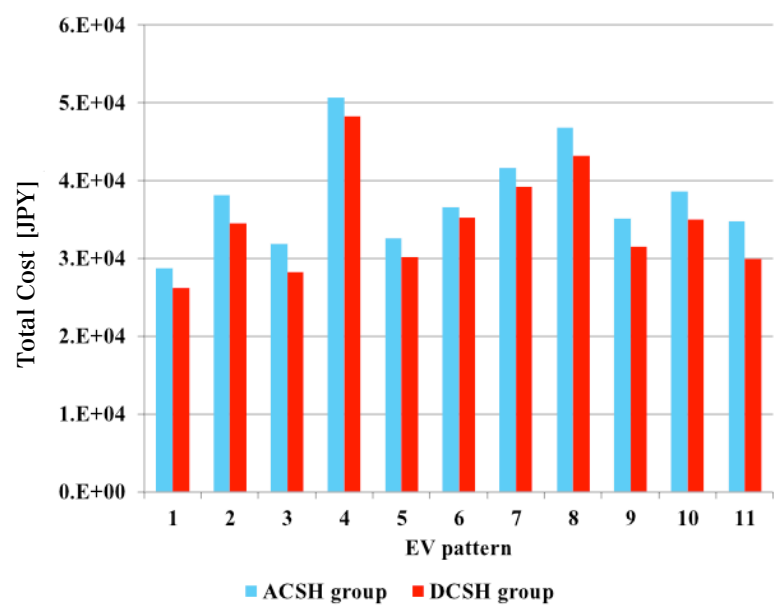

Figure 8: Total costs comparison with EV patterns.

is fixed at $40 \%$. Three EV operation patterns, going to the office/school, going shopping, and going on a trip are considered in this paper. All of the patterns are shown in Table 3. In case $\mathrm{A}$, an $\mathrm{EV}$ leaves the $\mathrm{SH}$ at 8:00 and returns at 19:00. It travels $40 \mathrm{~km}$ during the trip. In case B, an EV leaves the $\mathrm{SH}$ at 13:00 and returns at 17:00. It travels 15 $\mathrm{km}$ during the trip. In case C, an EV leaves the $\mathrm{SH}$ at 14:00 and returns at 22:00. It travels $160 \mathrm{~km}$ during the trip. After each trip, the EV charges to recover its SOC.

The total costs in each case are shown in Fig. 8. The costs for all patterns except case 1 increased because the EVs were charged after the trip. This pushed up the electrical demand and running cost of the SH. However, in every 
case, the costs of the DCSH group are lower than those of the ACSH group.

\section{Conclusion}

An economic evaluation of power supply forms in SHs, including EV operation patterns, was proposed in this paper. First, the effects of the DC load ratio were compared between two types of SH group. The DCSH group, which could share surplus power among SHs, realized lower costs than the ACSH group. Also, the effects of multiple EV operation patterns were evaluated. The DCSH could reduce the total costs in every pattern considered in this paper.

In future studies, a larger SH group will be considered and network construction costs will be calculated.

\section{Acknowledgment}

A part of this work was performed by Kazuki Narita, who graduated from the Advanced Course at the National Institute of Technology (KOSEN), Hakodate College.

\section{References}

[1] S. Gaurav, C. Birla, A. Lamba, S. Umashankar and S. Ganesan, "Energy Management of PV - Battery Based Microgrid System”, Procedia Technology, Vol.21, pp.103-111, 2015. DOI: $10.1016 /$ j.protcy.2015.10.016

[2] Y. Yoldas, A. Önen, S. M. Muyeen, A. V. Vasilakos and İ. Alana, "Enhancing smart grid with microgrids: Challenges and opportunities", Renewable and Sustainable Energy Reviews, Vol.72, pp.205-214, 2017. DOI: 10.1016/j.rser.2017.01.064

[3] I. Kurihara, "Power System Technology to Realize Smart Grid”, IEEJ Transactions on Power and Energy, Vol.133, No.4, pp.298-301, 2013(in Japanese).

[4] M. Shakeri, M. Shayestegan, S. M. Salim Reza, I. Yahya, B. Bais, M. Akhtaruzzaman, K. Sopiand and N. Amin, "Implementation of a novel home energy management system (HEMS) architecture with solar photovoltaic system as supplementary source", Renewable Energy, Vol.125, pp.108120, 2018. DOI: 10.1016/j.renene.2018.01.114

[5] H. Keiichi, "Trends of DC Power Technologies and their Applications", IEEJ Transactions on Power and Energy, Vol.131, No.4, pp.358-361, 2011. DOI: 10.1541/ieejpes. 131.358

[6] K. Tanaka, A. Yoza, K. Ogimi, A. Yona, T. Senjyu, T. Funabashi and C. H. Kim, "Optimal operation of DC smart house system by controllable loads based on smartgrid topology", Renewable Energy, Vol.39, pp.132-139, 2012. DOI: 10.1016/j.renene.2011.07.026

[7] K. Shimomachi, R. Hara and H. Kita, "Comparison between DC and AC Microgrid Systems Considering Ratio of DC Load", 2015 IEEE PES Asia-Pacific Power and Energy Engineering Conference (APPEEC), pp.1-4, 2015. DOI: 10.1109/APPEEC.2015.7380918

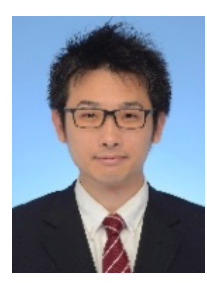

Kentaro Shimomachi (Member) was born in Hokkaido, Japan. He received a Ph.D. degree in engineering from Hokkaido University in 2016 and is presently an assistant professor at the $\mathrm{Na}$ tional Institute of Technology (KOSEN), Hakodate College. He has worked on power systems engineering, and he is member of the IEEJ and IEIEJ.

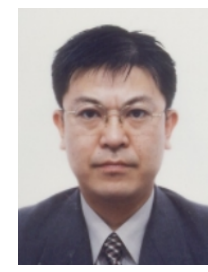

Yuji Mishima (Member) was born in Hokkaido, Japan. He received a Ph.D. degree in electrical engineering from Hokkaido University in 1999, and is presently a professor at the National Institute of Technology (KOSEN), Hakodate College. He has worked on power systems engineering, and he is member of the IEEJ and IEIEJ. 